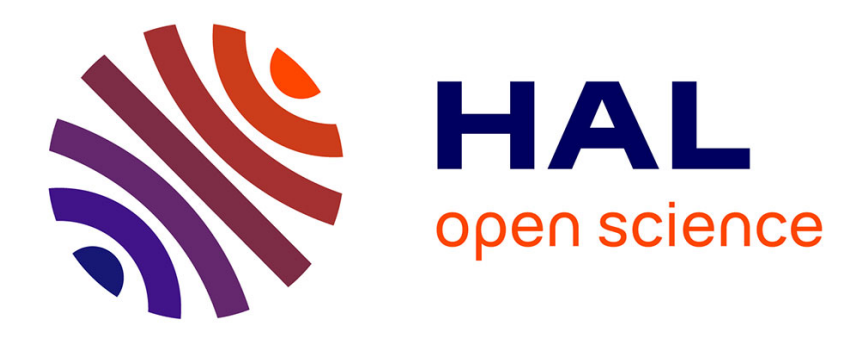

\title{
Viscous nonlinear theory of Richtmyer-Meshkov instability
}

Pierre Carlès, Stéphane Popinet

\section{To cite this version:}

Pierre Carlès, Stéphane Popinet. Viscous nonlinear theory of Richtmyer-Meshkov instability . Physics of Fluids, 2001, 13 (7), pp.1833-1836. 10.1063/1.1377863 . hal-01453024

\section{HAL Id: hal-01453024 \\ https://hal.science/hal-01453024}

Submitted on 7 Feb 2017

HAL is a multi-disciplinary open access archive for the deposit and dissemination of scientific research documents, whether they are published or not. The documents may come from teaching and research institutions in France or abroad, or from public or private research centers.
L'archive ouverte pluridisciplinaire HAL, est destinée au dépôt et à la diffusion de documents scientifiques de niveau recherche, publiés ou non, émanant des établissements d'enseignement et de recherche français ou étrangers, des laboratoires publics ou privés. 


\title{
Viscous non-linear theory of Richtmyer-Meshkov Instability
}

\author{
Pierre Carles and Stéphane Popinet \\ Laboratoire de Modélisation en Mécanique, Université Pierre et \\ Marie Curie, Case 162, 4 place Jussieu, 75252 Paris Cedex 05 (France)
}

(December 11, 2000)

\begin{abstract}
We propose a quantitative prediction of the effect of viscosity on the weakly non-linear impulsive Richtmyer-Meshkov instability between two fluids of arbitrary densities and viscosities. This theory is based on an asymptotic analysis of the Navier-Stokes equations using singular perturbation techniques. The law obtained for the interface deformation does not agree with former theoretical predictions of the effect of viscosity (Mikaelian, PRE 47, p.375 (1993)), but compares very well with direct numerical simulations we performed using a front-tracking code developed in our laboratory.
\end{abstract}


When a plane shock wave travels across an interface separating two fluids of different densities, all perturbations on the interface grow with time and a strong mixing of the two fluids ensues. This instability, named the Richtmyer-Meshkov (RM) instability (after it was theoretically predicted by Richtmyer [1] and experimentally observed by Meshkov [2]), appears in many different technical and scientific domains, including supersonic combustion, inertial confinement fusion and supernovae explosions. Very recently, a suggestion was made by Gueyffier and Zaleski [3,4] that the RM instability could also play an important role in impact phenomena between liquids and solids or liquids and liquids.

From a theoretical point of view, the RM instability can be approached in two ways: by linearizing Euler's equations for small interface perturbations around a base solution corresponding to the 1D propagation of a shock through a flat interface; or by assuming the fluids are incompressible and representing the passage of the shock by an impulsive acceleration perpendicular to the interface. The first approach has proved very efficient in predicting the maximum rate of growth of the perturbations for different initial conditions [1,5-7], but almost impossible to extend to the non-linear regime. On the other hand, the impulsive model for incompressible fluids is more appropriate for studying weak nonlinear effects $[8,9]$ and multi-mode coupling [10], but usually over-estimates the maximum growth rate. Recently, Zhang and Sohn [11] bridged the gap between the two approaches by using the linear compressible solution for describing the short-time evolution and a matched impulsive incompressible model for later times. Several authors in the past thirty years have discussed the limits of the impulsive model, often proposing methods for extending its validity to a larger range of parameters $[5,7,12-15]$.

In all these approaches however, viscous effects have been systematically disregarded, with the exception of a contribution by Mikaelian which we will discuss later [16]. Although viscous effects are often assumed to be negligible in most of the above-mentioned cases, this assumption is seldom based on quantitative arguments. The aim of this letter is to present a new, viscous, weakly non-linear theory of the RM instability enabling quantitative predictions of the intensity of viscous effects for two fluids of arbitrary densities and viscosi- 
ties. These predictions are compared to direct numerical simulations conducted with a full Navier-Stokes front-tracking code, and the excellent agreement which is obtained confirms the validity of the theory.

The theory is based on an asymptotic analysis of the Navier-Stokes equations written for two infinite incompressible viscous fluids separated by an interface with no surface tension (the effect of surface tension can be included in a very straightforward manner and will be discussed in more detail in a forthcoming paper). The fluids (noted 1 and 2) are initially at rest, the interface is slightly perturbed and an impulsive vertical acceleration is imposed in the 1-2 direction. Without viscosity, a discontinuity in the tangential velocity appears at the interface which subsequently becomes a vortex sheet. If viscosity is present, vorticity diffuses from the interface into the fluids and the growth rate of the instability is affected. In the present work, the viscous effects are taken into account in two boundary layers located on each side of the interface, in which equations analogous to Prandtl equations are treated. Far from the interface, inviscid equations are solved and the technique of Matched Asymptotic Expansions [17] is used to link the two systems in each fluid. Such a boundarylayer treatment imposes a restriction on the local Reynolds numbers in each fluid, which is always satisfied in experimental cases of practical interest (even in the case of liquid-liquid interfaces as in Jacobs and Sheeley's experiments [18]).

Let us suppose that the perturbed interface is initially defined by the equation:

$$
z=\eta(x, t=0)=\eta_{0} \cos (k x)
$$

(the $x$-axis is in the plane of the non-perturbed interface, the $z$-axis perpendicular to it and oriented in the opposite direction to that of the impulsive acceleration). The impulsive acceleration is defined as $g(t)=\Delta V \delta(t)$. In order to make an asymptotic expansion of the equations of the problem, they are first written in non-dimensional form, using the following normalizations (the superscript "( $i)$ " stands for fluid 1 or 2 ):

$$
x^{\prime}=x k, \quad z^{\prime}=z k, \quad t^{\prime}=\Delta V k t, \quad \eta^{\prime}=\frac{\eta}{\eta_{0}},
$$




$$
u^{(i) \prime}=\frac{u^{(i)}}{\Delta V}, \quad w^{(i) \prime}=\frac{w^{(i)}}{\Delta V}, \quad P^{(i) \prime}=\frac{P^{(i)}}{\left(\rho^{(1)}+\rho^{(2)}\right) \Delta V^{2}} .
$$

The non-dimensional equations then read as follows, omitting the primes (subscripts stand for partial derivatives):

$$
\begin{aligned}
u_{x}^{(i)}+w_{z}^{(i)} & =0 \\
u_{t}^{(i)}+u^{(i)} u_{x}^{(i)}+w^{(i)} u_{z}^{(i)} & =-\frac{\rho^{(1)}+\rho^{(2)}}{\rho^{(i)}} P_{x}^{(i)}+\frac{1}{\operatorname{Re}^{(i)}}\left(u_{x x}^{(i)}+u_{z z}^{(i)}\right), \\
w_{t}^{(i)}+w^{(i)} w_{x}^{(i)}+w^{(i)} w_{z}^{(i)} & =-\frac{\rho^{(1)}+\rho^{(2)}}{\rho^{(i)}} P_{z}^{(i)}+\frac{1}{\operatorname{Re}^{(i)}}\left(w_{x x}^{(i)}+w_{z z}^{(i)}\right)-\delta(t)
\end{aligned}
$$

with

$$
\stackrel{(i)}{\operatorname{Re}}=\frac{\Delta V \rho^{(i)}}{\mu^{(i)} k}
$$

where $\mu^{(i)}$ and $\rho^{(i)}$ are the viscosity and the density of fluid i. Far from the interface, the velocity vanishes and the pressure gradient becomes purely hydrostatic. At the interface (i.e. for $z^{\prime}=k \eta_{0} \eta^{\prime}$ ), the following boundary conditions hold:

$$
\begin{aligned}
u^{(1)} & =u^{(2)}, \quad w^{(1)}=w^{(2)}, \\
u_{z}^{(1)}+w_{x}^{(1)} & =\frac{\mu^{(2)}}{\mu^{(1)}}\left(u_{z}^{(2)}+w_{x}^{(2)}\right), \\
P^{(1)}-2 \frac{\rho^{(1)}}{\rho^{(1)}+\rho^{(2)}} \frac{1}{\operatorname{Re}^{(1)}} w_{z}^{(1)} & =P^{(2)}-2 \frac{\rho^{(2)}}{\rho^{(1)}+\rho^{(2)}} \frac{1}{\operatorname{Re}^{(2)}} w_{z}^{(2)} .
\end{aligned}
$$

Finally, the condition that the interface be a material surface reads (again for $z^{\prime}=k \eta_{0} \eta^{\prime}$ ):

$$
\eta_{t}=\frac{1}{k \eta_{0}} w^{(i)}-\eta_{x} u^{(i)}
$$

The usual condition of small initial perturbations is applied, which translates into: $\varepsilon=$ $k \eta_{0}<<1$. In terms of the non-dimensional $z^{\prime}$ variable, the thickness of the deformed interface is thus of order $\varepsilon$. In order to limit the viscous effects to the vicinity of the interface, the following condition is imposed: $\operatorname{Re}^{(i)}=O\left(\varepsilon^{-2}\right)$. A detailed asymptotic analysis shows that such a choice, among all the possible scalings for large $\mathrm{Re}^{(i)}$, leads to the most descriptive expansion. This conclusion is easy to reach on purely physical grounds: this choice indeed 
leads indeed to a boundary layer thickness of the same order as the interface deformation (i.e. $\varepsilon)$. For larger values of $\operatorname{Re}^{(i)}$, the boundary layer is smaller than the interface deformation and the curvature of the interface is neglected in the calculation of the viscous effects. For smaller values of $\operatorname{Re}^{(i)}$ on the contrary, the system in this thicker boundary layer degenerates, being unable to take into account the continuity of viscous stresses at the smaller scale of the interface. Hence, values of $\operatorname{Re}^{(i)}$ smaller or larger than $\varepsilon^{-2}$, provided they remain larger than 1 , lead to systems which are particular cases of the expansions obtained for $\operatorname{Re}^{(i)}$ of order $\varepsilon^{-2}$. Note that the condition that the Reynolds numbers $\operatorname{Re}^{(i)}$ be large compared to one is not a drastic one. Indeed, the velocity used to define these Reynolds numbers is not the average velocity of the fluid but the much larger velocity $\Delta V$ used in the definition of the impulsive acceleration $g(t)$. These "impulsive" Reynolds numbers are always very large in usual experiments (like in Ref. [18] or [19]).

Having defined all the non-dimensional quantities involved and their scaling with $\varepsilon$, it is now possible to construct asymptotic series for all the variables of the problem (i.e. $\eta$, $\left.u^{(i)}, w^{(i)}, P^{(i)}\right)$. The external systems (far from the interface) are obtained by making $\varepsilon$ tend to zero for $x^{\prime}$ and $z^{\prime}$ fixed. The boundary layer systems are obtained by making $\varepsilon$ tend to zero for $x^{\prime}$ and $\widetilde{z}^{\prime}$ fixed, with $\widetilde{z}^{\prime}$ the boundary layer space variable, defined as: $\widetilde{z}^{\prime}=z^{\prime} / \varepsilon$. The matching between external and boundary layers systems is obtained using a standard Matched Asymptotic Expansions technique. The pressure and velocity fields can be found by variable separation, solved in the physical space for external systems and in the Laplace space for the boundary layers. The inverse Laplace transforms of these solutions can be obtained analytically in the case with no surface tension and for the first two orders of expansion. This rather involved calculation will be detailed in a paper in preparation. In this letter, we only present the second-order expansion in $\varepsilon$ of the interface equation, obtained through this process:

$$
\eta^{\prime}\left(x^{\prime}, t^{\prime}\right)=\left(1+A t^{\prime}\right) \cos \left(x^{\prime}\right)-\varepsilon \frac{8 A}{3 \sqrt{\pi}} f\left(\rho^{(i)}, \mu^{(i)}\right) t^{\prime \frac{3}{2}} \cos \left(x^{\prime}\right)-\varepsilon \frac{1}{2} A^{3} t^{\prime 2} \cos \left(x^{\prime}\right)
$$

where $f$ is a function of the densities and the viscosities of the fluids, and $A$ the Atwood 
number, equal to $\left(\rho^{(1)}-\rho^{(2)}\right) /\left(\rho^{(1)}+\rho^{(2)}\right)$. When translated into dimensional form, this expansion gives the following viscous weakly non-linear law of interface deformation, the main result of this letter:

$$
\begin{aligned}
\frac{\eta}{\eta_{0}}(x, t)= & (1+A \Delta V k t) \cos (k x) \\
& -\frac{16}{3 \sqrt{\pi}} \frac{\sqrt{\rho^{(1)} \mu^{(1)}} \sqrt{\rho^{(2)} \mu^{(2)}}}{\left(\rho^{(1)}+\rho^{(2)}\right)\left(\sqrt{\rho^{(1)} \mu^{(1)}}+\sqrt{\rho^{(2)} \mu^{(2)}}\right)} A \Delta V k^{2} t^{\frac{3}{2}} \cos (k x) \\
& -\frac{1}{2} \eta_{0} A^{3} \Delta V^{2} k^{3} t^{2} \cos (2 k x) .
\end{aligned}
$$

The first term in the sum is the usual growth of the interface perturbation in the linear impulsive model; the second term represents the viscous attenuation of this growth; the third term is the first non-linear coupling of the perturbation with itself. Note that this third term is identical to the one obtained by Zhang and Sohn [8] or Berning and Rubenchik [10] in the non-viscous case. This identical non-linear term, apart from being an indirect validation of the whole calculation, shows that in the majority of cases (i.e. when the impulsive Reynolds numbers are large), viscous attenuation mainly affects the first deformation mode.

A third-order expansion in $\varepsilon$ would in turn give access to the second non-linear coupling of the perturbation with itself (with an $x$-dependence under the form of $\cos (k x)$ and $\cos (3 k x)$ ), together with the term representing the viscous attenuation of the first non-linear mode (in $\cos (2 k x))$. However, owing to the complexity of the calculation already at second order, such an expansion seems out of reach. Note that beside the interface deformation, the present calculation gives also access to the full velocity and pressure fields in the two fluids. This can be a very interesting information, for instance to study the evolution in time of the vorticity initially deposited at the interface by the shock (this point will also be discussed in the forthcoming article mentioned above).

In Ref. [16], Mikaelian proposed an expression for the linear RM instability between two viscous fluids. His method was based on moment equations applied to a non-viscous approximation of the vertical velocity, and lead to the following law of interface deformation: 


$$
\frac{\eta}{\eta_{0}}(x, t)=\left(1+A \Delta V \frac{1-e^{-2 k^{2} \nu t}}{2 k \nu}\right) \cos (k x) \quad \text { with } \quad \nu=\frac{\mu^{(1)}+\mu^{(2)}}{\rho^{(1)}+\rho^{(2)}}
$$

The expansion of this law for small times yields:

$$
\frac{\eta}{\eta_{0}}(x, t)=(1+A \Delta V k t) \cos (k x)-A \Delta V k^{3} \nu t^{2} \cos (k x),
$$

which should be compared to the first two terms of expression (1). The difference is both qualitative and quantitative: the power law in time is different, and for most cases the two expressions for the viscous correction differ by several orders of magnitude.

In order to check the validity of our theory, we have made a number of numerical simulations using a full Navier-Stokes incompressible front-tracking code developed by Popinet and Zaleski in recent years [20]. This code has been shown to give very accurate results, particularly in the case of the viscous damping of capillary waves. In the present study, two periodic layers of incompressible viscous fluids separated by a perturbed interface are initially superposed between two rigid boundaries. The distance between the boundaries is equal to the wavelength of the perturbation (using larger simulation domains does not change the results significantly, which shows that the effect of confinement is negligible). The domain is discretized using a regular cartesian grid $\left(256^{2}\right.$ cells) and an adaptive timestepping procedure is used to resolve the very short initial time scales. A strong acceleration is imposed for a very short time, and the response of the fluids is simulated. A Fourier transform of the shape of the interface is then calculated, and the first two terms (mode one and two) are compared with formula (1). Note that in order to increase precision in the simulation of the interface evolution, the dynamic viscosities of the fluids have been chosen equal (see Ref. [20,21]). The densities however are different, which leads to different kinematic viscosities.

The results obtained for a density ratio of $1 / 4$ in an unstable configuration $\left(\rho^{(1)}>\rho^{(2)}\right)$ and for several viscosities are presented in figures 1 and 2. Simulations with different density ratios have been performed. Similar results are obtained but are not detailed here. The first Fourier component (associated with $\cos (k x))$ minus the linear growth $(1+A \Delta V k t)$ is 
represented in figure 1 for different viscosities. The agreement between the numerical results and the predictions of Eq. (1) is very good. The viscous correction predicted by Mikaelian (i.e. Eq. (2) minus the linear growth $(1+A \Delta V k t))$ is also illustrated in figure 1 for the same series of Reynolds numbers. This comparison shows unambiguously that Eq. (2) is inappropriate.

The second Fourier mode (associated with $\cos (2 k x)$ ) is represented in figure 2. As viscosity decreases (large Reynolds numbers), the numerical result converges to the analytical one. The discrepancy between the theoretical and numerical curves can therefore clearly be attributed to the viscous attenuation of the growth of the second mode, not taken into account in the present theory. Note that this comparison is, to our knowledge, the first numerical confirmation of the validity of the first non-linear correction as calculated in Ref. [8] and [10] or in the present work.

In conclusion, we propose a quantitative prediction of the influence of viscosity on the RM instability, under the form of the interface deformation law given in Eq. (1). This theoretical law is in excellent agreement with the results of direct numerical simulations. Of course, the proposed asymptotic expansion is secular in time, so that it eventually fails after the interface has penetrated too deep into the non-linear regime. But the present theory remains nonetheless an important tool for two purposes: to estimate the influence of viscous effects on the RM instability, and in particular to provide quantitative arguments about the relevance of non-viscous theories; to serve as a benchmark for full Navier-Stokes numerical codes for two-phase flows, for which the Fourier analysis of the interface deformation obtained in the impulsive RM instability is a stringent test of precision.

The authors wish to acknowledge Dr. Denis Gueyffier for giving the initial motivation for this study. 


\section{REFERENCES}

[1] R.D. Richtmyer, Commun. Pure Appl. Math. 13, p.287 (1960).

[2] E.E. Meshkov, Izv. Akad. Nauk SSSR, Mekh. Zhidk. Gaza 5, p.151 (1969).

[3] D. Gueyffier, S. Zaleski, C.R. Acad. Sci. Paris 326, II b, p. 839 (1998).

[4] D. Gueyffier, PhD Thesis, Université Pierre et Marie Curie, Paris 6 (2000).

[5] Y. Yang, Q. Zhang, D. Sharp, Phys. Fluids 6, 5, p.1856 (1994).

[6] A.L. Velikovich, Phys. Fluids 8, 6, p.1666 (1996).

[7] J.G. Wouchuk and K. Nishihara, Phys. Plasmas 3, 10 (1996).

[8] Q. Zhang; S.I. Sohn, Physics Lett. A 212, p.149 (1996).

[9] A.L. Velikovich and G. Dimonte, Phys. Rev. Lett. 76, 17, p.3112 (1996).

[10] M. Berning and A.M. Rubenchik, Phys. Fluids 10, 7, p.1564 (1998).

[11] Q. Zhang and S.I. Sohn, Phys. Fluids 9, 4, p.1106 (1997).

[12] K.A. Meyer and P.J. Blewett, Phys. Fluids 15, 5, p.753 (1971).

[13] K.O. Mikaelian, Phys. Rev. Lett. 71, 18, p.2903 (1993).

[14] M. Vandeboomgaerde, C. Mügler, S. Gauthier, Phys. Rev. E 58, 2, p.1874 (1998).

[15] O.A. Hurricane, E. Burke, S. Maples, M. Viswanathan, Phys. Fluids 12, 8, p.2148 (2000).

[16] K.O. Mikaelian, Phys. Rev. E 47, 1, p.375 (1993).

[17] J. Kevorkian and J.D. Cole, Perturbation methods in applied mathematics, SpringerVerlag (1981).

[18] J.W. Jacobs and M. Sheeley, Phys. Fluids 8, 2, p.405 (1996). 
[19] M. Vetter and B. Sturtevant, Shock Waves 4, p.247 (1995).

[20] S. Popinet and S. Zaleski, Int. J. Numer. Meth. Fluids 30, p.775 (1999).

[21] S. Popinet, PhD Thesis, Université Pierre et Marie Curie, Paris 6 (2000). 


\section{FIGURES}

FIG. 1. Viscous correction to the linear growth based on Eq. (1), Eq. (2) (Mikaelian [16]) and numerical simulations for several Reynolds numbers.

FIG. 2. Non-linear correction to the linear growth based on Eq. (1) and numerical simulations for several Reynolds numbers. 


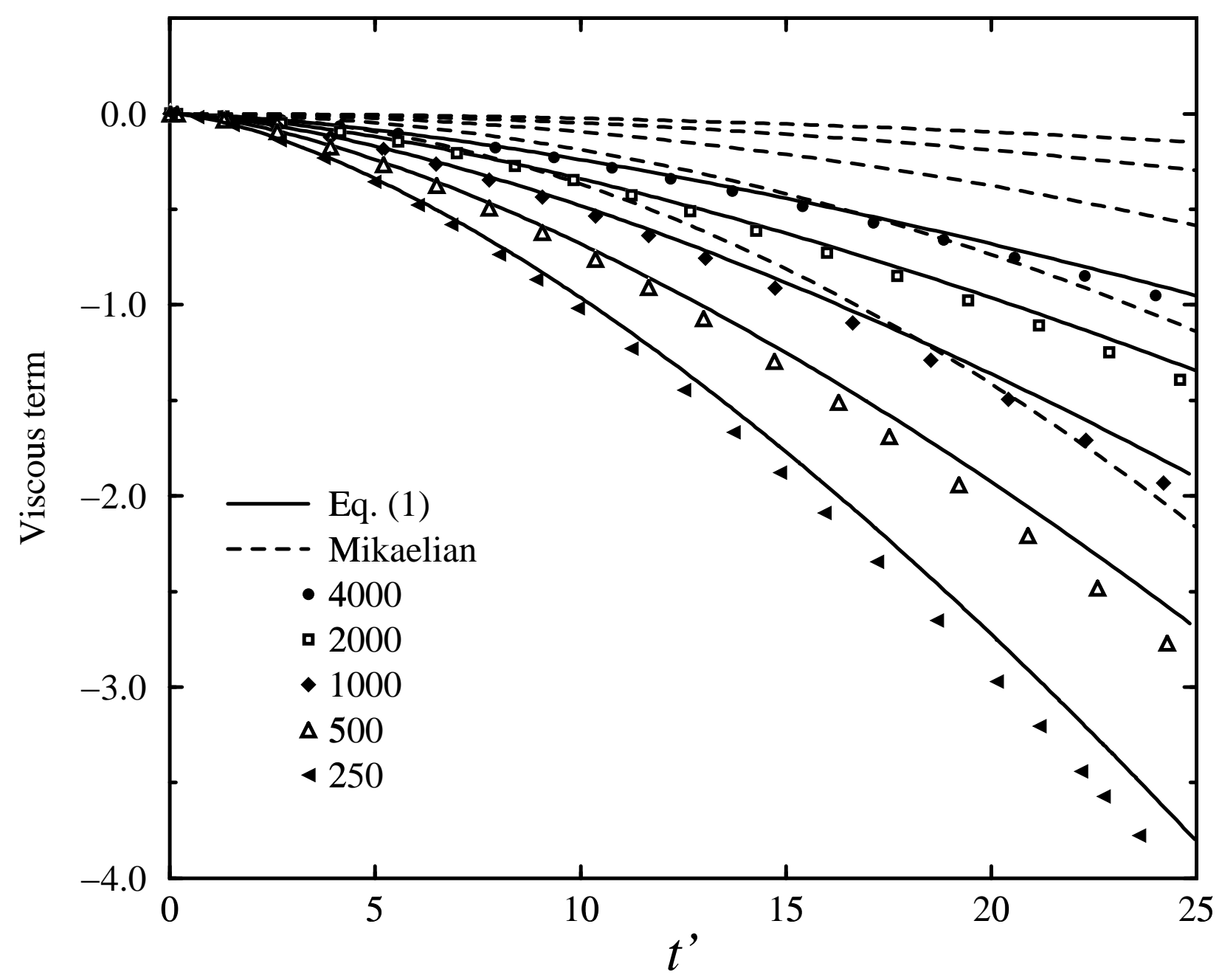

Figure 1 


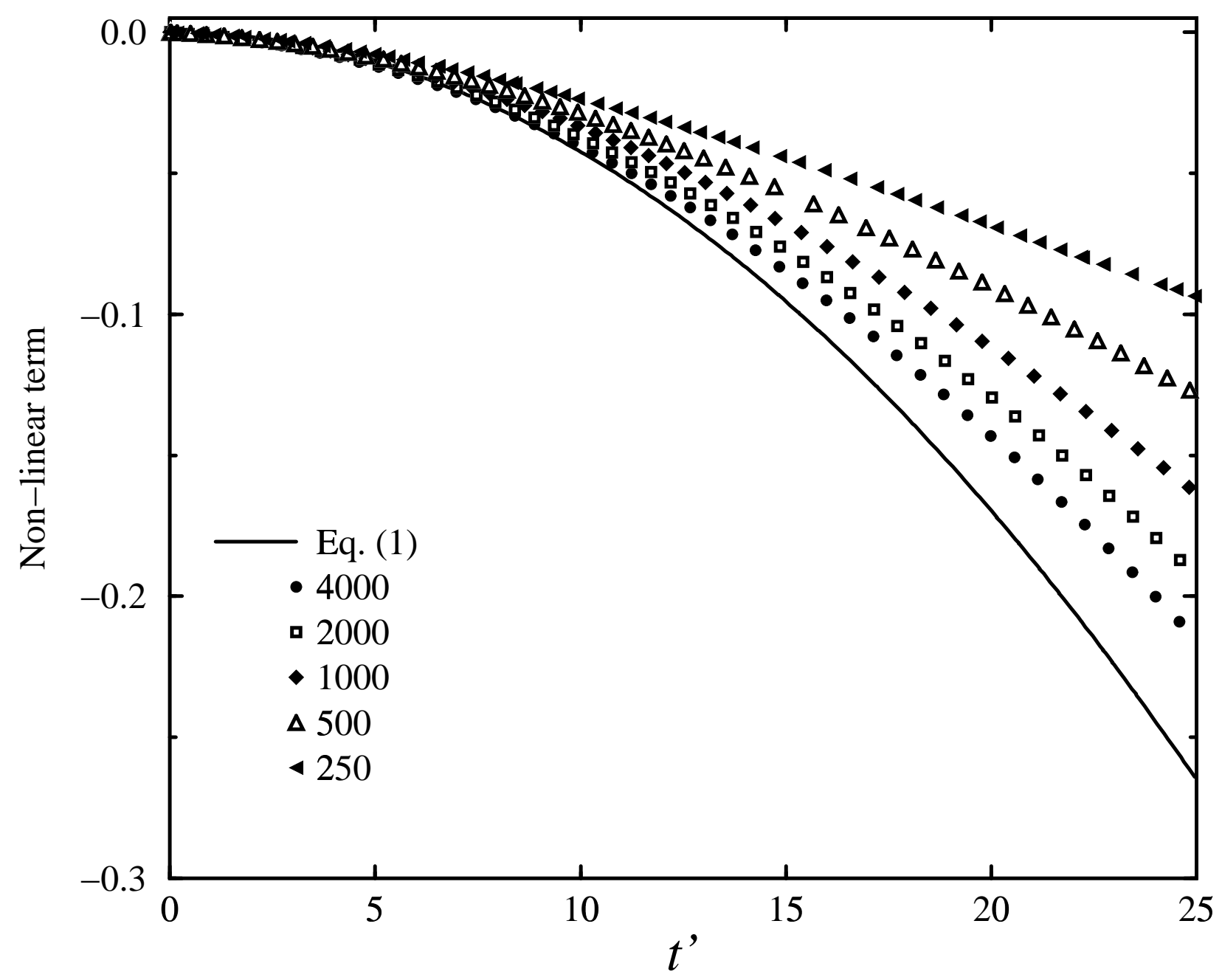

Figure 2 